\title{
Controlled Evaporative Assembly of Polymers from Confined Solutions
}

\author{
ZHIQUN LIN \\ Department of Materials Science and Engineering, lowa State University, Ames, lowa 50011
}

Received 3 July 2010; revised 28 July 2010; accepted 28 July 2010

DOI: $10.1002 /$ polb.22136

Published online 20 September 2010 in Wiley Online Library (wileyonlinelibrary.com).

KEYWORDS: confined geometries; confined solutions; controlled evaporative assembly; ordered structures; self-assembly; structure; surfaces

INTRODUCTION In the process of drying, nonvolatile solutes (e.g., polymers, proteins, viruses, bacteria, DNA, microspheres, nanocrystals, carbon nanotubes, etc.) contained within a sessile droplet (i.e., unconstrained liquid) readily assemble into a diverse range of intriguing one- or two-dimensional structures, possessing dimensions of a few hundred submicrons and beyond. It is, in principle, a nonequilibrium process. ${ }^{1}$ Two main characteristic patterns are often observed: "coffee rings" and irregular network structures (i.e., Benard cells). A "coffee ring" forms when, in the absence of natural convection and surface tension gradients, the contact line of an evaporating drop becomes pinned. This ensures that liquid evaporating from the edge is replenished by liquid from the interior, so the outward flow carries the nonvolatile dispersions to the edge. $^{2}$ The evaporation flux varies spatially, with the highest flux observed at the edge of drop. A subset of the "coffee rings" phenomenon is the formation of concentric rings by repeated microscopic pinning and depinning events (i.e., "stick-slip" motion) of the three-phase contact line,, that is, the competition between the friction force and surface tension of the solution. However, random concentric rings are generally formed. Moreover, based on Navier-Stokes equations with a lubrication approximation, the bulk of current theoretical work has centered on understanding a single ring formation using either analytical ${ }^{5,6}$ or numerical methods. ${ }^{7,8}$ By contrast, only a few elegant theoretical studies have focused, either analytically $^{3}$ or numerically, ${ }^{9}$ on the formation of periodic multirings (i.e., concentric rings) during droplet evaporation on a substrate. A gradient of temperature normal to the droplet surface due to solvent evaporation can induce a Marangoni-Benard convection (i.e., closed-loop circular convection), ${ }^{10-13}$ which results in irregular Benard cells caused by the upward flow of lower, warmer liquid. The spatial variation of evaporative flux and possible convection mean, however, that these nonequilibrium, dissipative structures (e.g., "coffee rings," ${ }^{2}$ cellular structures, ${ }^{11}$ fingering instabilities, ${ }^{14}$ etc.) are often irregular and stochastically organized.

In this context, to take advantage of the extreme simplicity of this nonlithographic, external field-free, top-down tech- nique and exploit its full potential for developing miniaturized optical, electronic, optoelectronic, magnetic devices, and so forth, control of the evaporative flux, solution concentration, and interfacial interactions among the solvent, solute, and substrate is necessary to construct highly ordered, complex structures rapidly and cheaply over large areas. To date, a few impressive studies have centered on the precise manipulation of the evaporation process, including controlled anisotropic wetting/dewetting processes ${ }^{15,16}$ controlled dewetting by dip-coating, ${ }^{17}$ convective assembly in evaporating menisci, ${ }^{18}$ evaporation-induced assembly in restricted geometries, ${ }^{19-29}$ and evaporative lithography using a mask, ${ }^{30,31}$ to create complex deposit patterns with unprecedented regularity.

Notably, evaporative assembly has been applied to a wide spectrum of inorganic nanomaterials of different types, sizes, and shapes. In the latter context, it has been demonstrated that solutions of nanoparticles (NP), nanorods, disk-like particles, spherocylinders, asymmetric dimers, tetrapods, and even a binary mixture of two distinctively different kinds and shapes of crystals (e.g., Au NP with CdSe nanorods) have readily assembled into a variety of intriguing regular structures upon irreversible solvent evaporation. ${ }^{1,32}$ However, we make no attempt to cover this literature. Moreover, the drying of unconstrained polymer solution that leads to ordered structures will also not be discussed here; this includes seminal work on breath figure templated assembly of polymer films by Francois ${ }^{33}$ and Srinivasarao. ${ }^{34}$ For detail on this subject the reader is referred to a more comprehensive review. ${ }^{35}$ Finally, in this Perspective, surface reconstruction of thin block copolymer films by exposure to selective solvent vapor, regardless of single solvent, mixed solvents, or solvent/water involved, to achieve long range vertical and lateral orderings (i.e., evaporative ordering) is not considered as evaporative assembly from solutions, and thus will not be covered. Instead, this Perspective seeks to highlight several approaches based on drying-mediated assembly of polymers (e.g., latex particles, homopolymers, block copolymers, etc.) that yield complex ordered structures by subjecting polymer 
solutions to evaporate in confined geometries (i.e., constrained solutions).

\section{Evaporation of Confined Polymer Solutions}

To date, several elegant approaches have been successfully exploited to control droplet evaporation in confined geometries, which include two plates with one plate placed at a certain angle against another horizontal substrate, ${ }^{18,36}$ two parallel plates with the top one sliding on the lower stationary substrate, ${ }^{19,20}$ cylindrical tube, ${ }^{28,29}$ two crossed cylindrical mounts covered with single crystals of mica sheets, ${ }^{21}$ and curve-on-flat geometries ${ }^{22-26,37,38}$ as illustrated in Figure 1. The confined geometries provide a unique environment for remarkable control over the flow within an evaporating droplet, which, in turn, regulates structure formation.

Controlled, rapid deposition of structured coatings from latex colloidal crystal suspensions is enabled by dragging at a constant velocity a small amount of aqueous polymer suspension confined in a meniscus between two plates placed on one another at certain angles [Fig. 1(a)]. ${ }^{18,36}$ The governing mechanism of crystal deposition is convective assembly at high volume fractions. The coating thickness (i.e., the number of particle layers) and structure are controlled by deposition speed and particle volume fraction [Fig. 2(a)]. ${ }^{18}$ Compared to conventional dip-coating, the coating time (from hours or days to minutes) and materials consumption (single surface of the substrate as opposed to both sides of the substrate) are markedly decreased.

When the polymer solution is constrained between two parallel plates [Fig. 1(b)], controlling the speed of the upper sliding plate allows self-organized, mesoscale polymer patterns (e.g., dots, stripes, and ladders) ${ }^{19}$ to form continuously at the receding meniscus on the stationary lower substrate [Fig. 2(b)]. Furthermore, hierarchical assembly can be achieved using self-assembled block copolymers as nonvolatile solutes. ${ }^{20}$ The size and shape of polymer patterns are highly reproducible and dictated by processing parameters such as the sliding speed and polymer concentration. Higher sliding speed and lower concentration lead to slower evaporation rates, while slower sliding speed and higher concentration result in faster evaporation rates. ${ }^{19}$

Drying a solution of latex particles or bio-NP in a cylindrical capillary placed vertically ${ }^{28}$ or horizontally ${ }^{29}$ yields bands of particle aggregates along the capillary [Fig. 1(c)]. The radius of curvature of the meniscus and the associated capillary force are dictated by the radius of the capillary, which is constant and much smaller than the capillary length. The use of a capillary also provides a confined space that effectively regulates the evaporation rate of the fluidic column, which in turn impacts the kinetics of colloidal crystallization. ${ }^{28}$ The band width and spacing along the capillary increase with an increase of colloidal concentration or with a decrease in time at a given initial concentration [Fig. 2(c)].

Constraining a drop of polymer solution in a confined geometry composed of a curved upper surface on a flat substrate (i.e., curve-on-flat geometry) leads to a capillary-held solution [Fig. 1(d)]. Because of imposed geometrical constraint, the evaporation is restricted to occur only at the capillary edge. ${ }^{22,23}$ As a result, the controlled, repetitive pinning and depinning of the three-phase contact line yields a lateral surface morphology naturally and spontaneously that consists of hundreds of highly ordered concentric polymer deposits with regular spacing [Fig. 2(d)]. Other ordered yet complex structures including spokes, fingers, serpentines, and hierarchically organized structures can also be produced. ${ }^{22-27,37-39}$ Each deposit is a few to tens of nanometers high and submicron to several microns wide.

It is noteworthy that, in addition to the aforementioned methods to manipulate evaporative deposition of polymers, recently a novel approach termed evaporative lithography was utilized to pattern colloidal films. ${ }^{30,31}$ The polymer nanoparticle solution is dried beneath a mask that induces periodic variations between regions of hindered and free evaporation. The colloidal patterns can be regulated by varying the separation distance between the mask and underlying film and the mask geometry.

\section{Parameters Tailored}

Although the concentration effect has been widely investigated in copious previous work, a number of additional parameters should be delicately tailored to provide detailed insight into the structure formation produced by controlled evaporative assembly.

\section{Solvent Effect}

Slow solvent evaporation suppresses instabilities, thereby leading to regular patterns. Rapid evaporation increases the convective force due to evaporative cooling, thereby forming convection cells, fractal branches, fingering instabilities, and so forth. ${ }^{11}$ Notably, the use of mixed solvents may trigger heterogenous evaporation of solutions, which in turn results in intriguing complex structures in confined geometries.

\section{Molecular Weight Effect}

When the molecular weight of polymers is low, the viscosity of the solution front is so low that no contact line will be pinned, leading to the rupture of a liquid-like thin film (i.e., dewetting) and, eventually, the formation of stochastic structures.

\section{Chemical Structure Effect}

Rather than glassy amorphous polymers, the use of polymers with different chemical natures, for example, semicrystalline polymers and conjugated polymers (e.g., regioregular poly(alkyl thiophene)) can influence the competition between the evaporative assembly and crystallization/orientation, which may yield anisotropic structures, for example, regular or irregular microscopic patterns composed of periodically or randomly oriented nanowires/nanofibrils or nanorods.

\section{Composition Effect}

When an evaporating droplet contains two or more nonvolatile solutes, for example, a binary polymer blend, a mixture of block copolymer/NP (i.e., polymer A-b-polymer B/polymer A-functionalized NP), a ternary mixture of polymer blend/NP (i.e., polymer A/polymer B/polymer A-functionalized NP), or even multicomponent suspensions, the synergy of phase 
(a)

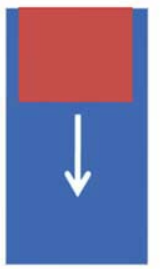

Top View

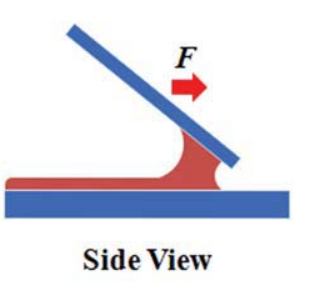

Side View

(c)

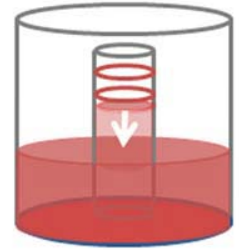

Side View (3D)

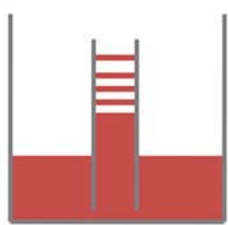

Side View (b)
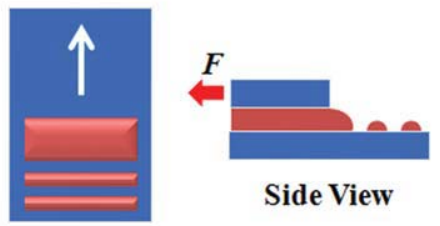

Side View

Top View (d)

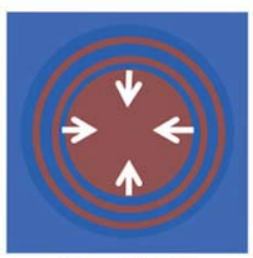

Top View

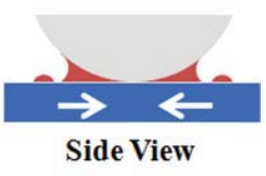

Side View

FIGURE 1 (a) Convective assembly of colloidal particles. A drop of colloidal suspensions was confined between two glass plates, that is, a lower sample substrate and an upper tilted plate, which meet at certain angle, forming a wedge-like capillary edge. (b) Convective self-organization of mesoscale polymer patterns from an evaporating polymer solution confined between two parallel plates. Control over the speed of the upper sliding plate allows patterns to form continuously at the receding meniscus on the stationary lower substrate. (c) Cylindrical tube-assisted assembly process. A glass cylindrical capillary is placed vertically in an open glass bottle containing a solution of volatile solvent and nonvolatile solute such as colloidal particles or polymers. (d) Controlled evaporative assembly of a confined microfluid in a curve-on-flat geometry. A wide variety of well ordered structures (e.g., concentric "coffee rings," rectangles, pyramids, triangular contour lines, fishnets, and so forth can be achieved by judicious choice of the solution concentration, shape of the upper curved surface, polymers, and so on.

(a)

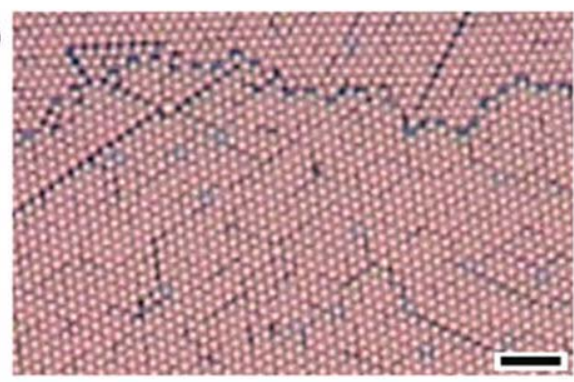

(c)

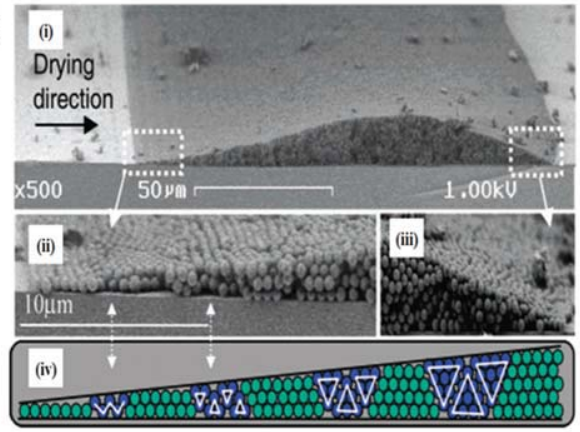

(b)

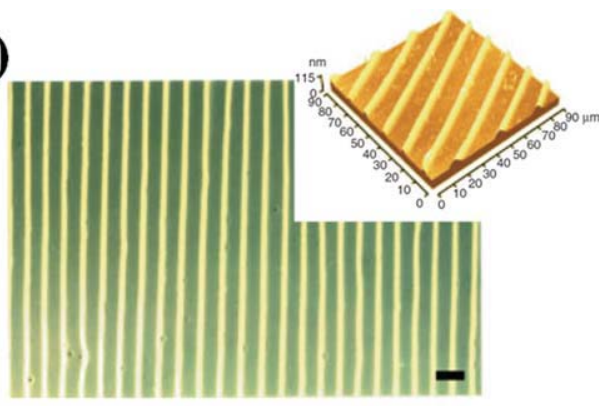

(d)

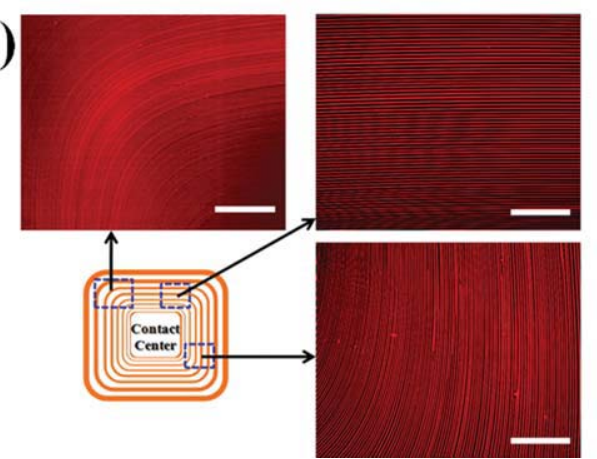

FIGURE 2 (a) Convective assembly of polystyrene latex particle monolayer at natural assembly speed. ${ }^{18}$ Scale bar $=5 \mu \mathrm{m}$. Reproduced from ref. 18, with permission from The American Chemical Society, Copyright 2004. (b) Convective self-organization of poly(3hexylthiophene) (P3HT) stripes from an evaporating P3HT chloroform solution confined between two glass plates. ${ }^{19}$ Reproduced from ref. 19, with permission from Wiley-VCH Verlag GmbH \& Co. KGaA, Copyright 2005. (c) SEM images of a band structure in the capillary. ${ }^{28}$ (i) One band with the drying direction indicated. Two close-ups show the smooth structure at the beginning (ii) and the end (iii) of the band, respectively. (iv) Schematic of the typical succession of the hexagonal close-packed regions. Reproduced from ref. 28, with permission from The American Chemical Society, Copyright 2004. (d) Representative fluorescence micrographs of concentric square stripes formed from controlled evaporative assembly of poly[2-methoxy-5-(2-ethylhexyloxy)-1,4-phenylenevinylene] (MEH-PPV) toluene solution in a pyramid-on-flat geometry. ${ }^{25}$ Scale bars are $600 \mu \mathrm{m}$ in the upper left and $300 \mu \mathrm{m}$ in the upper and lower right images. Reproduced from ref. 25, with permission from Wiley-VCH Verlag GmbH \& Co. KGaA, Copyright 2009. 
separation of polymer blends, coassembly of surface-modified NPs and block copolymers to allow preferential segregation of NPs within a target domain, and the destabilization of polymer mediated by the interfacial interaction between the polymer and the substrate during the course of solvent evaporation in confined geometries, can lead to the formation of appealing complex structures.

\section{Geometry Effect}

The variation of the separation distance between two plates or the tilting angle of the upper plate will inevitably influence the solvent evaporation rate and the associated capillary flow, leading to the formation of different morphologies. For example, in the case of evaporative assembly in curveon-flat geometries [Fig. 1(d)], faster solvent evaporation resulting from the smaller radius of curvature of the upper surface may induce homogeneous deposition, intermediate evaporation may cause the pinning and depinning of the contact line to yield "coffee rings," and very slow evaporation could transport the solutes to the curve/flat contact center.

\section{Surface Chemistry Effect (Chemical Heterogeneity)}

The surface hydrophobicity of confined geometries, which is related to the interfacial interaction between the solute and the substrate (i.e., interfacial interaction effect or substrate effect), will predictably govern structure formation. By tuning the interfacial interaction between the polymer and the substrate that governs the stability of liquid thin films, the ordered dissipative structures may be produced as a result of the synergy of controlled assembly of the polymer and its destabilization mediated by the interfacial interaction. The surface of substrates in confined geometries are hydrophilic as they are typically made of silica-based materials. Thus, studies can also be performed on a droplet of solution confined between adjoining hydrophobic and hydrophilic surfaces (i.e., a Janus interface). To accomplish this, a selfassembled hydrophobic monolayer can be deposited to render one surface hydrophobic while keeping the other surface hydrophilic.

\section{Patterned Surface Effect (Physical Heterogeneity)}

The use of a topographically patterned surface in confined geometries, for example, square-shaped pillars, circular posts, parallel trenches, and so forth on the lower flat substrate, may offer a unique capability of tuning the local capillary flow of polymer solution, thereby inducing polymer chains, such as DNA and conjugated polymer nanowires, to stretch, align, and immobilize in or around the patterns to form compelling self-assembled structures. The combination of physically patterned surfaces with chemical modification may result in an even richer family of unexpected structures.

\section{Effect of the Shape of Confined Geometry}

A wide range of compelling periodic polymer assemblies (e.g., ellipses, triangles, squares, polygons, etc.) would be possible by simply tailoring the shape of the upper surface of confined geometries [Fig. 1(d)] and of the capillary [Fig. 1 (c)] to guide the evaporation process.

\section{External Field Effect}

(a) External perturbation: (1) Pump vertically (i.e., hydrodynamic drainage): Pumping is achieved by periodically moving the upper surface at proper amplitude and frequency while keeping the lower surface stationary, thereby squeezing fluid out of (or into) the intervening gap [e.g., Fig. 1(d); or accomplished by moving the cylindrical capillary in Fig. 1(c)]. Pumping generates a symmetric flow that may direct structure formation radially, which is analogous to the dynamic combing of DNA. ${ }^{40}$ (2) Shear laterally: Unidirectional oscillatory shear at proper amplitude and frequency can be applied, which may either perturb or impart structure formation by modifying the flow unidirectionally. (b) External electric or magnetic field: An electric or magnetic field applied across the polymer solution in conjunction with the solvent evaporative field may assist the assembly of polymers, making the deposition process more rapid, reducing the number of defects in the structures, ${ }^{36}$ and potentially achieving improved orientation and ordering of structures, especially the long range ordering of nanodomains when block copolymers or block copolymer/NP mixtures are employed as nonvolatile solutes.

\section{Temperature Effect}

Heating the lower surface while keeping the upper surface cool would impose a temperature gradient, $\Delta T$, or vice versa. The magnitude of $\Delta T$ can be varied. The $\Delta T$-induced Marangoni-Benard convection ${ }^{10-13}$ is expected to affect controlled solvent evaporation, thereby forming intriguing structures.

\section{Challenges}

Despite the recent progress on controlled evaporative assembly, many scientific issues related to evaporation-induced flow and deposition still remain. Some of them are described as follows.

\section{Theory and Simulation}

Some phenomena involved in structure formation from a sessile drying droplet can be understood and modeled through a basic transport analysis of evaporation and evaporation-induced flow, using either a lubrication approximation to achieve an analytical solution or numerical analysis. Likewise, the ability to predict the length scales of periodicity, height, and width, and then compare them with experimental observations, is key to understanding the mechanisms of structure formation via controlled evaporative assembly in confined geometries. Although models of evaporating pure liquid in a capillary tube or on parallel glass plates have been developed, ${ }^{41-45}$ few theoretical studies have been performed on a solution containing nonvolatile solute evaporating in confined geometries. The theory, modeling, and computer simulations on evaporation of confined microfluids and its induced flow should be developed to provide a basis for fully understanding the evaporative assembly in confined geometries.

\section{Real Time Visulation}

The flow field and associated particle transport in an evaporating droplet can be visualized using the tool of particle image velocimetry to help understand the solute transport, 
in which the use of micron-sized particles is necessary. To map the flow patterns inside an evaporating droplet containing polymer chains that may self-assemble into nanostructures or crystallize during the solvent evaporation, it is crucial to fully utilize current or develop new optical techniques that can offer reliable and appropriate measurement. For example, fluorescence dynamics techniques image the trajectory of polymer chains and in situ polarized Raman spectroscopy techniques monitor the Raman shift as a function of time to provide information on structural anisotropy. Moreover, it would be invaluable to exploit spectroscopic techniques to study the real time phase separation, assembly, and crystallization that occur concurrently in the proximity of the moving three-phase contact lines. This remains a challenge.

\section{Three-Dimensional Assembly}

Current state-of-art evaporative assembly methods are limited to the preparation of $2 \mathrm{D}$ ordered structures. However, it is highly desirable to create 3D structures for many technological applications such as photonic crystals, electronics, MEMS, and so forth. New approaches based on extremely simple evaporative assembly to rationally "synthesize" 3D polymer structures should be designed and developed. One route that may lead to 3D assembly and ordering of polymer structures is to use prepatterned substrates, ${ }^{46}$ either physically or both chemically and physically prepatterned, to direct the transport of polymer particles to fill the patterns or situate in the vicinity of patterns.

\section{CONCLUSIONS}

The significance of controlled evaporative assembly in confined geometries lies in its promise to lead to a new paradigm for creating highly ordered structures on surfaces over large areas in a simple, precisely controllable, and cost-effective manner, dispensing with the need for lithographic techniques. The scope of potential applications for such structures is vast, encompassing combinatorial chemistry, electronics, optical coatings, optical materials, photonics, optoelectronics, microfluidic devices, magnetic materials, information processing and data storage devices, nanotechnology, sensors, DNA/RNA microarrays, gene mapping of DNA, high-throughput drug discovery, and biotechnology. This topic is extraordinarily rich. The future of controlled evaporative assembly is bright and appears to be only limited, at present, by the imagination of innovative methods to control the evaporation process and associated capillary flow to craft a wider spectrum of complex ordered structures.

The author gratefully acknowledge the support from the National Science Foundation (NSF CBET-0844084 and NSF CMMI-0968656).

\section{REFERENCES AND NOTES}

1 Rabani, E.; Reichman, D. R.; Geissler, P. L.; Brus, L. E. Nature 2003, 426, 271.

2 Deegan, R. D.; Bakajin, O.; Dupont, T. F.; Huber, G.; Nagel, S. R.; Witten, T. A. Nature 1997, 389, 827.
3 Adachi, E.; Dimitrov, A. S.; Nagayama, K. Langmuir 1995, 11, 1057.

4 Shmuylovich, L.; Shen, A. Q.; Stone, H. A. Langmuir 2002, $18,3441$.

5 Deegan, R. D.; Bakajin, O.; Dupont, T. F.; Huber, G.; Nagel, S. R.; Witten, T. A. Phys Rev E 2000, 62, 756.

6 Popov, Y. O. Phys Rev E 2005, 71, 036313.

7 Fisher, B. J. Langmuir 2002, 18, 60.

8 Ozawa, K.; Nishitani, E.; Doi, M. Jpn J Appl Phys 2005, 44, 4229.

9 Nonomura, M.; Kobayashi, R.; Nishiura, Y.; Shimomura, M. J Phys Soc Jpn 2003, 72, 2468.

10 Maillard, M.; Motte, L.; Pileni, M. P. Adv Mater 2001, 13, 200.

11 Nguyen, V. X.; Stebe, K. J. Phys Rev Lett 2002, 88, 164501.

$12 \mathrm{Hu}, \mathrm{H} . ;$

13 Hu, H.; Larson, R. G. J Phys Chem B 2006, 110, 7090.

14 Pauliac-Vaujour, E.; Stannard, A.; Martin, C. P.; Blunt, M. O.; Notingher, I.; Moriarty, P. J.; Vancea, I.; Thiele, U. Phys Rev Lett 2008, 100, 176102.

15 Gleiche, M.; Chi, L. F.; Fuchs, H. Nature 2000, 403, 173.

16 Chen, X.; Rogach, A. L.; Talapin, D. V.; Fuchs, H.; Chi, L. F. J Am Chem Soc 2006, 128, 9592.

17 Huang, J.; Kim, F.; Tao, A. R.; Connor, S.; Yang, P. D. Nature Mater 2005, 4, 896.

18 Prevo, B. G.; Velev, O. D. Langmuir 2004, 20, 2099.

19 Yabu, H.; Shimomura, M. Adv Funct Mater 2005, 15, 575.

20 Kim, B. H.; Shin, D. O.; Jeong, S.-J.; Koo, C. M.; Jeon, S. C.; Hwang, W. J.; Lee, S.; Lee, M. G.; Kim, S. O. Adv Mater 2008, 20, 2303

21 Lin, Z. Q.; Granick, S. J Am Chem Soc 2005, 127, 2816.

22 Hong, S. W.; Xu, J.; Xia, J.; Lin, Z. Q.; Qiu, F.; Yang, Y. L. Chem Mater 2005, 17, 6223.

$23 \mathrm{Xu}$, J.; Xia, J.; Hong, S. W.; Lin, Z. Q.; Qiu, F.; Yang, Y. L. Phys Rev Lett 2006, 96, 066104.

24 Xu, J.; Xia, J.; Lin, Z. Q. Angew Chem Int Ed 2007, 46, 1860.

25 Hong, S. W.; Byun, M.; Lin, Z. Q. Angew Chem Int Ed 2009, $48,512$.

26 Hong, S. W.; Wang, J.; Lin, Z. Q. Angew Chem Int Ed 2009, 48, 8356 .

27 Byun, M.; Bowden, N. B.; Lin, Z. Q. Nano Lett 2010, 10, 3111.

28 Abkarian, M.; Nunes, J.; Stone, H. A. J Am Chem Soc 2004, $126,5978$.

29 Lin, Y.; Balizan, E.; Lee, L. A.; Niu, Z.; Wang, Q. Angew Chem Int Ed 2010, 49, 868.

30 Harris, D. J.; Hu, H.; Conrad, J. C.; Lewis, J. A. Phys Rev Lett 2007, 98, 148301.

31 Harris, D. J.; Lewis, J. A. Langmuir 2008, 24, 3681.

32 Bigioni, T. P.; Lin, X. M.; Nguyen, T. T.; Corwin, E. I.; Witten, T. A.; Jaeger, H. M. Nature Mater 2006, 5, 265. 
33 Widawski, G.; Rawiso, M.; Francois, B. Nature 1994, 369 387.

34 Srinivasarao, M.; Collings, D.; Philips, A.; Patel, S. Science 2001, 292, 79.

35 Bunz, U. H. F. Adv Mater 2006, 18, 973.

36 Kleinert, J.; Kim, S.; Velev, O. D. Langmuir 2010, 26, 10380.

37 Hong, S. W.; Xia, J.; Lin, Z. Q. Adv Mater 2007, 19, 1413.

38 Hong, S. W.; Xia, J.; Byun, M.; Zou, Q.; Lin, Z. Q. Macromolecules 2007, 40, 2831.

39 Byun, M.; Laskowski, R. L.; He, M.; Qiu, F.; Jeffries-EL, M.; Lin, Z. Q. Soft Matter 2009, 5, 1583.
40 Michalet, X.; Ekong, R.; Fougerousse, F.; Rousseaux, S.; Schurra, C.; Hornigold, N.; van Slegtenhorst, M.; Wolfe, J.; Povey, S.; Beckmann, J. S.; Bensimon, A. Science 1997, 277, 1518.

41 Khrustalev, D.; Faghri, A. J Heat Transfer 1996, 118, 725.

42 Bennacer, R.; Sefiane, K.; El-Ganaoui, M.; Buffone, C. Int J Numer Meth Heat Fluid Flow 2003, 14, 877.

43 Clement, F.; Leng, J. Langmuir 2004, 20, 6538.

44 Schaffer, E.; Wong, P. Phys Rev E 2000, 61, 5257.

45 Stange, M.; Dreyer, M. E.; Rath, H. J Phys Fluids 2003, 15, 2587.

46 Choi, S.; Stassi, S.; Pisano, A. P.; Zohd, T. I. Langmuir 2010, 26, 11690. 